\title{
PARAMETRIC ACCURACY: BUILDING INFORMATION MODELING PROCESS APPLIED TO THE CULTURAL HERITAGE PRESERVATION
}

\author{
S. Garagnani ${ }^{\text {a, } *}$, A. M. Manferdini ${ }^{\text {a }}$ \\ a Department of Architecture DA, University of Bologna (simone.garagnani, am.manferdini)@unibo.it
}

Commission V, WG V/4

KEY WORDS: BIM, laser scanning, AEC digital tools, Autodesk Revit, GreenSpider plugin.

\begin{abstract}
:
Since their introduction, modeling tools aimed to architectural design evolved in today's "digital multi-purpose drawing boards" based on enhanced parametric elements able to originate whole buildings within virtual environments. Semantic splitting and elements topology are features that allow objects to be "intelligent" (i.e. self-aware of what kind of element they are and with whom they can interact), representing this way basics of Building Information Modeling (BIM), a coordinated, consistent and always up to date workflow improved in order to reach higher quality, reliability and cost reductions all over the design process. Even if BIM was originally intended for new architectures, its attitude to store semantic inter-related information can be successfully applied to existing buildings as well, especially if they deserve particular care such as Cultural Heritage sites. BIM engines can easily manage simple parametric geometries, collapsing them to standard primitives connected through hierarchical relationships: however, when components are generated by existing morphologies, for example acquiring point clouds by digital photogrammetry or laser scanning equipment, complex abstractions have to be introduced while remodeling elements by hand, since automatic feature extraction in available software is still not effective. In order to introduce a methodology destined to process point cloud data in a BIM environment with high accuracy, this paper describes some experiences on monumental sites documentation, generated through a plug-in written for Autodesk Revit and codenamed GreenSpider after its capability to layout points in space as if they were nodes of an ideal cobweb.
\end{abstract}

\section{INTRODUCTION}

\subsection{BIM origins and Cultural Heritage sites}

Notoriously, CAD systems were meant to offer the possibility of simultaneous design production as well as integrated specifications, blurring the distinction between designers and drafters. Sophisticated CAD tools focused on architectural design started to spread in the AEC professional practices beginning from last two decades of the past century, replacing traditional drawings on paper with digital ones (Bermudez and King, 2000). Ever since, the computer drafting and modeling concept has shift in its paradigm, evolving from sort of a "multi-purpose drawing boards" to complete design frameworks based on enhanced parametric objects, combined together to originate whole buildings within virtual environments.

This relatively new approach implies that every object introduced is not only a geometric representation of real shapes or technical components, as in CAD systems, but it's a data collector on its own, with specific meanings to the architect or the project manager.

These elements provide an abstract computer representation of a physical world that is convenient for designers: a wall intended as an object, represents an actual wall in the physical world as well as a door as a digital object represents a real door, maybe connected to the previous wall.

This advanced graphic duality is essential in design validation and it is considered the basic of Building Information Modeling (BIM), a coordinated, consistent and always up to date working process strongly supported by several software tools, continuously improved in order to reach higher quality, reliability, optimized scheduling, errors and costs reduction together with avoidance of any possible project misinterpretation (Garagnani, Cinti Luciani and Mingucci, 2011). Therefore, BIM has not to be considered only as a set of software to produce drafts and models but, on the contrary, as a pipelined process among designers, managers, engineers, architects and contractors, sharing a common language on a framework at their disposal (Eastman, Teicholz, Sacks and Liston, 2011).

This way, BIM becomes a collaborative strategy in architectural design, in which the term "information" implies a sense of transparency among actors, in order to generate a team working culture devoted to efficiency and integration, not only focused on software mastering.

Even computer programs explicitly written for BIM purposes evolved their interface from traditional GUI to a modern objectoriented one, highlighting their ease of use to guide designers attention on projects and not on tools.

Modern BIM environments, in fact, can easily create and manage parametric geometries, collapsing them in primitives that can be connected one by one through hierarchical relationships: this is a flawless procedure when creating simple objects from software libraries, where shapes and constraints are already set, but it's much more difficult when components are generated to represent heterogeneous and original existing morphologies, for example acquiring data using digital photogrammetry or laser scanning equipment.

In this case BIM abstractions have to be mostly re-modeled by hand, since automatic feature recognition in available commercial AEC computer programs are still not effective, experimental and under development by several software houses.

\footnotetext{
* Corresponding author.
} 
This aspect is amplified dealing with historic monuments, mainly made of components and materials whose geometry and characteristics are very far from specifications of modern software libraries.

However, BIM process and paradigm would represent a significant step into much more sophisticated methodologies aimed at Cultural Heritage documentation.

A possible solution to the lack of geometric primitives issue, experimented and documented in scientific literature, involves the so called Historic Building Information Modeling (HBIM) approach, in which parametric objects are built beginning from historic data (surveys, assays and literature) and layered in plugin libraries aimed to map the elements onto point clouds data (Dore and Murphy, 2012).

This methodology, unfortunately, assumes buildings to be documented through correctly codified components, already present in libraries, with limited capabilities to change them in order to represent possible existing different states.

This is why terrestrial laser scanning (TLS), for example, gained interest in architectural documentation, even more since these technologies are becoming cheaper than in the past: this kind of high definition survey tools can be steadily adopted by practices due to its stability and well documented effectiveness. Of course similar considerations can be extended to digital photogrammetry, since photography has long been recognized as the most important form of documentation, especially in case of historic buildings; nowadays dense point clouds extraction from pictures is speeded up greatly by computer vision software, with evident benefits in $3 \mathrm{~d}$ reconstructions.

\subsection{High definition surveys and BIM process}

Several professionals and academic studies on point clouds translation into graphic primitives have been developed for advanced development in the field of buildings digital reconstruction, even in real time (Früh, Jain and Zakhor, 2005).

Numerous algorithms have been proposed for either interpolating or approximating an unorganized, noisy set of points on a surface, in order to obtain simpler parametric geometries. These developments were motivated by the consideration that primitive objects are, of course, lighter and much more editable in computer memory than their respective original master dataset.

A parametric approach, in fact, ties geometry together with values able to describe elements through their numeric dimension or their mutual, physical constraints (in this case parametric modeling becomes variational).

This kind of "existing buildings reverse engineering" require that $3 \mathrm{~d}$ models are ultimately intended for human manipulation. This is not only due to their essence of "as built" documents; but it implies the need for accurate inference of geometric and topological information that can be expressed in terms of component features and interconnections.

Nevertheless, a critical aspect in TLS applications, as generally in computer vision techniques too, is the correct segmentation of elements, since it is still hard to automatically tell walls from windows or ceilings from floors, especially considering transparent surfaces.

However, acquiring geometries remotely and turning them into objects abstractions, would amplify the model capability to store information, also considering various typologies of elements.

High precision survey on materials deterioration for example, followed by data recording into BIM components, allows database productions where information can be filtered accordingly to operators needs with specific duties in restorations or renovations, preparing a powerful documentation system able to memorize data for future interventions.

This is particularly useful dealing with Cultural Heritage preservation in monumental sites (Fai, Graham, Duckworth, Wood and Attar, 2011), a field of studies in which the need for not ambiguous and durable database systems is extremely important. In this case monument documentation is intended as a process aimed to collect data by storing topological information and content relationships in order to output to several devices, from analytical software engines to paper sheets or mobile viewers such as PDAs or tablet PCs.

Autodesk Revit for example, a widely used software in most of the practices all over the world, was one of the first BIM-related computer program to adopt point clouds import plug-ins in order to reach this archiving versatility since its 2012 release (Garagnani and Cinti Luciani, 2011). Initially imagined as an aid to re-modeling buildings, Autodesk's current efforts are focused on point cloud features extraction improvement, especially aimed at automatic floors and walls recognition beginning from selected point regions.

The technology preview available on the Autodesk Labs website is interesting, even if some glitches and bugs have still to be fixed.

Once registered (i.e. referenced) one another, point clouds can be merged to approximate planes and single parametric elements can be generated by mesh interpolation, even considering thousands of vertexes. In both cases file transfer format from raw laser scanner data have to be carefully verified in order not to loose information due to excessive decimation or bad manifold translation.

Semantic splitting and real elements topologies are not transferred when importing point clouds in BIM modelers so metadata that permit object to be "smart" or "intelligent" (i.e. equipped with a sort of self-consciousness of what kind of element they are and with whom they can interact) have to be specified later (De Luca, 2012).

This is the point where human operators can introduce their knowledge in proper component segmentation, especially in historic building documentation, where actors who produce digital models could be historians and archaeologists, not only traditional AEC professionals.

\section{SOME EXPERIENCES ON CULTURAL HERITAGE SITES}

Some practical tests, aimed at fine tuning a working methodology for data gathering by means of photogrammetry or TLS surveys, were conducted to process dense point clouds in a BIM environment.

Sant'Apollinare Nuovo in Ravenna, Italy, one of the most important examples of early Christian Byzantine art and architecture in Western Europe was a noticeable case study, due to his importance and historic legacy. Sant'Apollinare Nuovo was erected by Theodoric as his palace chapel during the first quarter of the VI century A.D., with an apse and atrium typical of ancient Basilicas, with side walls covered with mosaics.

Measures were acquired using a Leica ScanStation C5 TOF laser scanner survey, and then integrated with some dense point surfaces obtained through digital photogrammetry, using Menci Z-Scan Software.

The final result was a model of the inner nave, including some details captured with a higher number of points: point clouds were superimposed in order to get a complete documentation of walls and mosaics (Figure 1). 

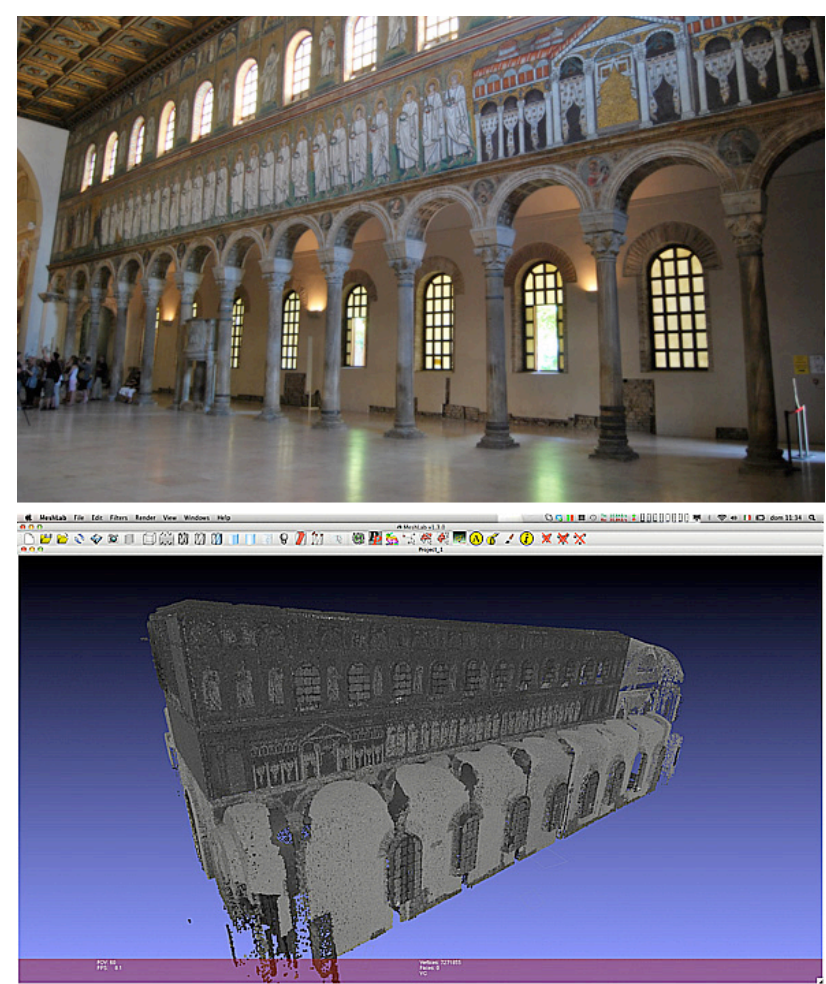

Figure 1. Sant'Apollinare Nuovo: the early Byzantine church is famous worldwide for its nave surrounded by walls covered with mosaics (top image). Some 3D laser scanner and digital photogrammetric surveys were performed in order to obtain a registered point cloud of interiors (bottom image).

Afterwards, point clouds were registered, decimated and processed in order to transform them in proper geometric input for Autodesk Revit Architecture 2012.

Decimation was an indispensible practice, as a matter of fact, scan files often reach values of hundreds of millions to billions of points hosted, with datasets including homogeneous and unsegmented elements, so that computer hardware resources have to be huge in order to allow points editing and to parameterize the whole sets in the BIM environments.

The simplified dataset was transferred using .PLY file format, even if Revit standard import procedure of point cloud automatically converts them into .PCG format.

The original idea was to parametrically remodel the entire building mostly by hand, keeping laser survey and photogrammetric data as a sort of scaffolding for smart objects assembly.

Even if some commercial plug-ins aimed to point clouds importing and editing in Revit exist, such as Leica CloudWorx or Imaginit Scan to BIM, they weren't used to favour standard workflows.

Autodesk's automatic feature recognition plug-in, introduced in the first quarter of 2012, could not be tested since it is not yet available.

The usual pipeline consists in point cloud importing (for efficiency and improved performance reasons, Revit uses only a limited subset of points for display and selection at any given time), in visualization in work area with linear units check, and in the generation of Revit families starting from visual data, if smart object are required instead of a frozen set of points.

In this environment, visualized point clouds cannot be used directly as snap points; geometry creation or modification tools can snap to implicit planar surfaces instead, which are dynamically detected in the point cloud. However, Revit only detects planes that are perpendicular to the current work plane (in plan, elevation, or 3D) and only in a small proximity of the cursor. This is not so useful for users in need to approximate segmented geometries in order to translate them into smart objects.

\subsection{The developed application}

Due to this occurrences, a simple plug-in was developed with the ambition to implement a versatile tool able to import vertexes of interest only from the cloud, considering them as discrete, selectable snap points to reproduce accurate components with precise extensions, with the application of a completely different workflow.

This plug-in, codenamed GreenSpider, has the capability to layout points in space as if they were nodes of an ideal cobweb. It consists in a Microsoft Windows .NET 3.5 Dynamic-link library (DLL) developed in C\# by means of RevitAPI 2012; this code is able to conveniently parse ASCII text files containing $\mathrm{XYZ}$ coordinates derived from real world data capture, translating them into native reference points in Revit's mass modeling environment.

Using this tool, the parametric production of smart elements is easier and much more accurate (Figure 2).

Once set up through an installer that can be freely downloaded from the Internet (http://www.tcproject.net/pivotx/? $e=149$ ), GreenSpider can be accessed directly in Revit graphical user interface (GUI) from an icon placed in the external plug-ins ribbon. Afterwards, the code searches for a file with .GSP extension, a plain text file format where $\mathrm{XYZ}$ numerical values representing vertexes are separated by semicolons, while commas are used for decimal marks.

This plug-in operates metric conversion for Revit Architecture; as a consequence, .GSP values are originally required in centimetres.

GreenSpider is still a prototype so it's possible to run only two commands identified by two different icons in Revit's GUI: GSpoints and GScurves.

The first one imports the vertex point cloud, while the second traces a spline interpolation among imported vertexes, in order to build surfaces with accuracy.

This way the plug-in allows an easier modeling capability to Revit, which considers points as vertexes of simple parametric primitives pertaining to native families. The generated shapes can therefore be imported in master models as smart masses, with semantic metadata embedded, allowing hyperlink presence to multimedia contents, self-conscious topology, lighter memory consumption and so on.

This plug-in was tested upon other laser scanner data pertaining ancient buildings.

In those case studies, point clouds were firstly sectioned using planar surfaces in order to reduce the number of information without losing singularities.

Each section generated a single ASCII file that was then imported in Revit, following a semantic criterion.

Afterwards, GScurve option was used to connect vertexes through splines.

Parametric elements were then built interpolating these edges. The selected case studies exemplify how complex point clouds can be simplified through planar sections and can subsequently be imported by means of a semi-automatic methodology, as it requires the intervention of an operator in order to select the most appropriate location of section planes able to reduce complexity without losing edges, nor singularities (Figure 3). 

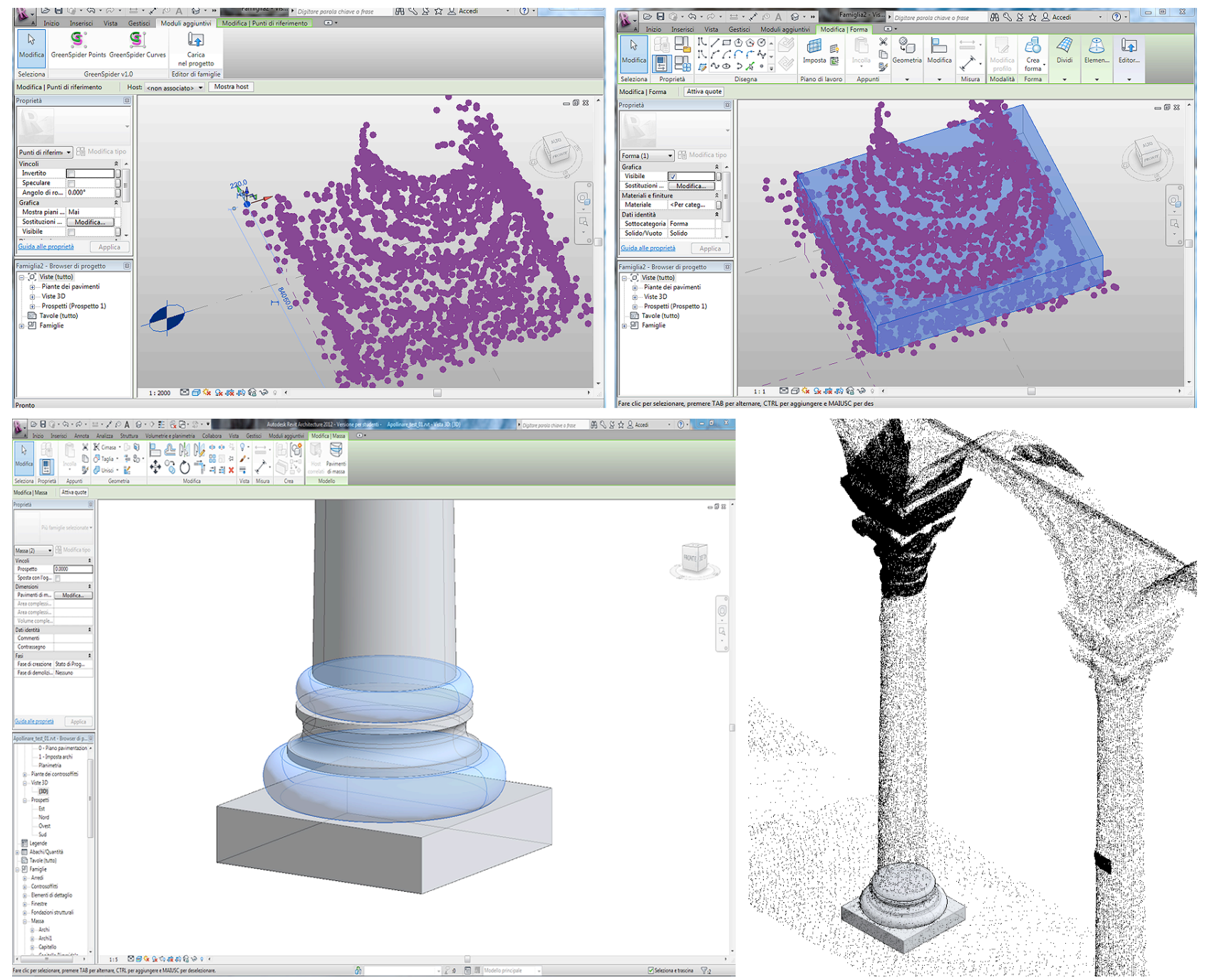

Figure 2. GreenSpider plug-in for Autodesk Revit 2012, developed by S. Garagnani in order to import selected vertexes from unstructured, relevant point clouds as native reference points. This way "smart" BIM elements can be traced out with accuracy obtaining parametric objects, which can be semantically segmented.

\section{CONCLUSIONS}

Building Information Modeling is a novel strategy intended to optimize the building design process, avoiding among other things spatial conflicts, time wasting reworks and costs associated with them.

Even if it's been developed for new buildings, the typical BIM attitude to gather data about components and relate it by virtue of their self-awareness and semantics, can be successfully applied to existing architectures as well.

This is a considerable aspect since the database of contents hosted in a digital model that is structured following BIM criteria is suitable to document heterogeneous Cultural Heritage sites.

In order to be as faithful as possible to reality, these contexts have to be surveyed using high definition technologies that are destined to capture data about morphologies and state of preservation of elements.
Dense point clouds are credited as a valid digital substitute aimed at representing reality with accuracy. They can be used as output from active TLS or passive photogrammetric techniques. Computer $3 \mathrm{~d}$ models that are generated this way can be useful for documentation purposes, for periodic checks on structures preservation and for studies about technological building systems over architecture history.

However, point clouds are not structured entities, they are recorded without topology, nor semantic discretization.

On the contrary, BIM requires well-defined relationships among components, called "smart" by virtue of their knowledge about their mutual placement.

Importing un-structured datasets into BIM software is still a tricky process but some progresses can be made using dedicated plug-ins, such as GreenSpider for Revit. This application is able to translate points into native reference snaps aimed to allow elements modeling with much more precision instead of a simple tracing out. 

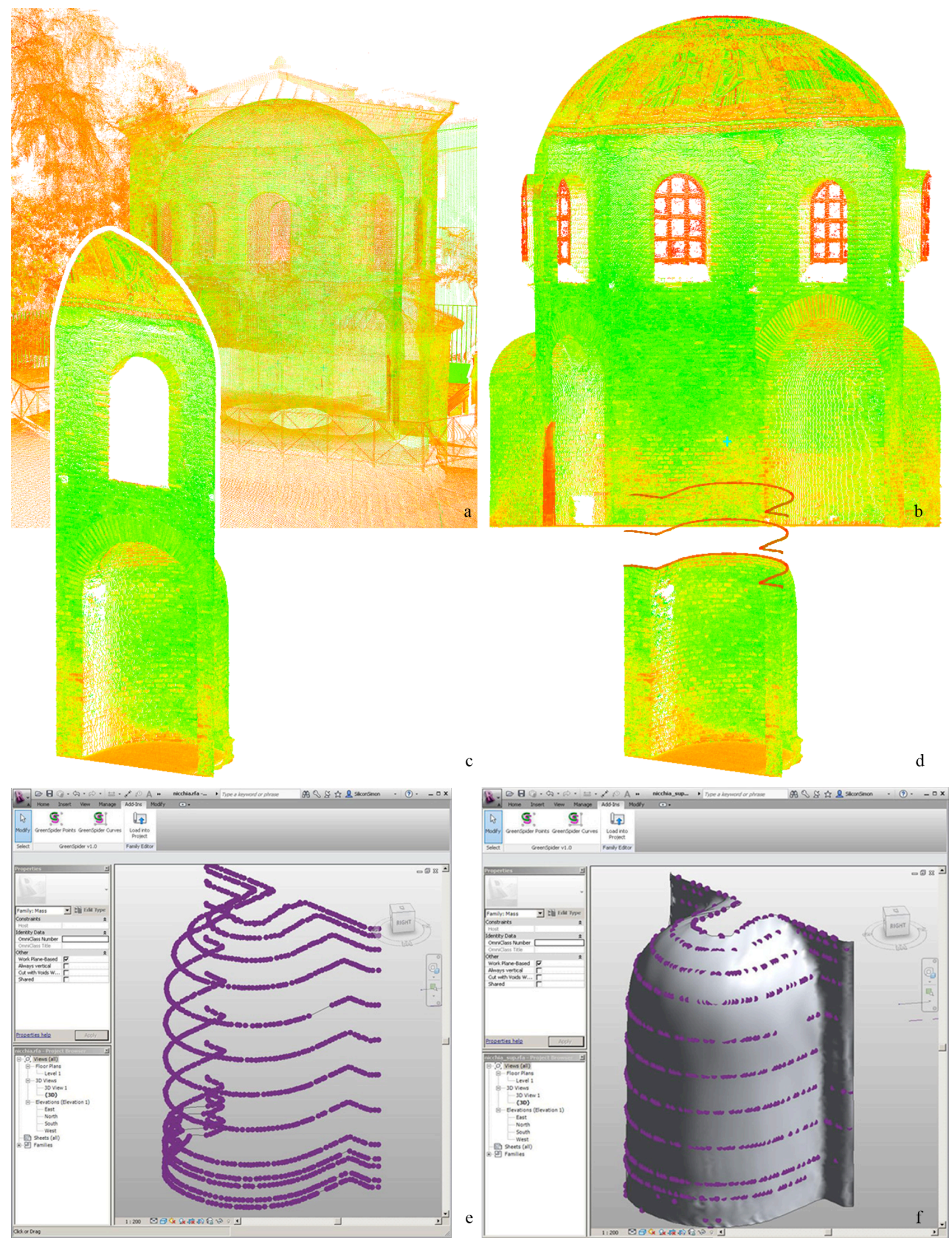

Figure 3. The adopted pipeline. A complex point cloud (a) is firstly segmented in its main entities (b) and single elements (c). These last geometries are then sectioned (d) in order to drastically reduce geometric complexity and meanwhile preserve singularities that are indispensable for the following 3d reconstruction in the BIM environment. Through the use of the developed GreenSpider application, these data are then imported into the Revit Architecture (e). The snappable sets of points are then connected through curves (e) and afterwards inter-connected using parametric entities (f). 
The novelty of this process relies on the integration between BIM environment and reality-based 3D data.

As a matter of fact, the developed GreenSpider plug-in allows managing point clouds as entities that can be used as inputs in the building of parametric elements process. As a consequence, if compared with other tools that are actually available, the derived parametric geometry better fits the surveyed realitybased geometry.

As presented in this paper, a dedicate BIM database for complex historic architectures can therefore be generated in order to gather information and make it available for researchers, professionals or generic actors involved in Cultural Heritage preservation.

\section{ACKNOWLEDGEMENTS}

GreenSpider is a free Revit 2012 plugin written by S. Garagnani, under a Creative Commons Attribution-Non Commercial - Share Alike 3.0 Unported (CC BY-NC-SA 3.0) license. GreenSpider can be freely downloaded at http://www.tcproject.net.

The authors would like to thank Dr. Franco Faranda (MIBAC Ministero per $i$ Beni e le Attività Culturali, Italian ministry of heritage and cultural activities) for his willingness to allow access and surveys in Sant'Apollinare Nuovo.

The author would also like to thank Mariangela Vandini who is representative of the research team who develops the "Protocols for the enhancement, conservation and fruition of architectonical cultural heritage" at the CIRI (Centro Interdipartimentale di Ricerca Industriale) of the University of Bologna, for her precious collaboration that was indispensable to conduct investigations on the case study of the Battistero degli Ariani in Ravenna.

The research has been held thanks to the equipment made available by Roberto Mingucci, Scientific Responsible of the Silab Laboratory of the Department of Architecture of the University of Bologna. The authors would like to thank also Giovanni Bacci of the Silab Laboratory for his precious support during survey operations.

\section{REFERENCES}

Bermudez, J., King, K., 2000. Media interaction and design process: Establishing a knowledge base. In Automation in Construction, 9, pp. 37-56.

Garagnani, S., Cinti Luciani, S., Mingucci, R., 2011. Building Information Modeling: la tecnologia digitale al servizio del progetto di architettura. In DISEGNARECON, 4(7), pp. 5-19.

Eastman, C. M., Teicholz, P., Sacks, R., Liston, K., 2011. BIM Handbook. A Guide to Building Information Modeling for Owners, Managers, Designers, Engineers, and Contractors. John Wiley \& Sons.

Dore, C., Murphy, M., 2012. Integration of Historic Building Information Modeling and 3D GIS for Recording and Managing Cultural Heritage Sites. In VSMM2012. Proceedings of the 18th International Conference on Virtual Systems and Multimedia. Virtual Systems in the Information Society, pp. 369-376.

Früh, C., Jain, S. and Zakhor, A., 2005. Data Processing Algorithms for Generating Textured 3D Building Facade Meshes from Laser Scans and Camera Images. In International Journal of Computer Vision, 61 (2), pp. 159-184.
Fai, S., Graham, K., Duckworth, T., Wood, N., Attar, R., 2011. Building Information Modelling and Heritage Documentation. In Proceedings of XXIII CIPA International Symposium, Prague, Czech Republic, 12/16 September 2011.

Garagnani, S., Cinti Luciani, S., 2011. Il modello parametrico in architettura: la tecnologia B.I.M. di Autodesk Revit. In DISEGNARECON, 4(7), pp. 20-29.

De Luca, L., 2012. Methods, Formalisms and Tools for the Semantic-Based Surveying and Representation of Architectural Heritage. Applied Geomatics, 1866-9298, pp. 1-25. 